\title{
A Low-Cost Through-Wall FMCW Radar for Stand-Off Operation and Activity Detection
}

\author{
Kevin. Chetty*a, Qingchao Chen ${ }^{\mathrm{a}}$, Matthew Ritchie ${ }^{\mathrm{b}}$, Karl Woodbridge ${ }^{\mathrm{b}}$ \\ ${ }^{a}$ Dept of Security and Crime Science, University College London. 35 Tavistock Square. WC1H9EZ; \\ ${ }^{b}$ Dept. of Electronic \& Electrical Engineering, University College London. Torrington Pl. WC1E7JE \\ *k.chetty@ucl.ac.uk; phone +44 (0)20 31083169
}

\begin{abstract}
In this paper we present a new through-wall (TW) FMCW radar system. The architecture of the radar enables both high sensitivity and range resolutions of $<1.5 \mathrm{~m}$. Moreover, the radar employs moving target indication (MTI) signal processing to remove the problematic primary wall reflection, allowing higher signal-to- noise and signal-to-interference ratios, which can be traded-off for increased operational stand-off. The TW radar operates at $5.8 \mathrm{GHz}$ with a $200 \mathrm{MHz}$ bandwidth. Its dual-frequency design minimises interference from signal leakage, and permits a baseband output after deramping which is digitized using an inexpensive 24-bit off-the-shelf sound card. The system is therefore an order of magnitude lower in cost than competitor ultrawideband (UWB) TW systems. The high sensitivity afforded by this wide dynamic range has allowed us to develop a wall removal technique whereby high-order digital filters provide a flexible means of MTI filtering based on the phases of the returned echoes. Experimental data demonstrates through-wall detection of individuals and groups of people in various scenarios. Target positions were located to within $\pm 1.25 \mathrm{~m}$ in range, allowing us distinguish between two closely separated targets. Furthermore, at $8.5 \mathrm{~m}$ standoff, our wall removal technique can recover target responses that would have otherwise been masked by the primary wall reflection, thus increasing the stand-off capability of the radar. Using phase processing, our experimental data also reveals a clear difference in the micro-Doppler signatures across various types of everyday actions.
\end{abstract}

Keywords: Through-wall radar, FMCW radar, micro-Doppler, moving target indication.

\section{INTRODUCTION}

\subsection{Through-the-Wall Radar for Safety and Security}

The ability to detect the presence of individuals behind visually obscuring structures is of significant benefit to police, security agencies and emergency services. Through-Wall (TW) radar technology may improve tactical and operational capability during emergencies ranging from terrorist and hostage events, through to building collapse and fire. In tactical situations, first responders from the police and security services require a high level of situational awareness to perform their duties safely and effectively.

Currently, there are a limited number of commercial TW systems available to law enforcement and the emergency services. Cost, deployment and user operability issues have resulted in relatively limited exploitation of this technology worldwide. In domestic security applications the technology has also raised concerns about privacy and inappropriate use $^{1}$ which need to be addressed when brought into operation.

There is a significant body of academic research ${ }^{2,3}$ which has attempted to apply radar detection and imaging techniques ${ }^{4}$ to scenarios that consist of an obscuring wall barrier. The main objectives in these research studies has been to facilitate methods and techniques that permit: the identification of objects and people; characterisation of behavioural activities; and mapping the structural layout of interiors ${ }^{5}$. TW radar systems have by no means reached their full potential thus there is still a requirement for improvements in the technology to enhance their utility for existing applications in security, surveillance and search \& rescue, as well as to find new and novel applications. To that end many of the challenges which arise in TW detection scenarios have been, and continue to be addressed by the academic research community.

Radar Sensor Technology XXI, edited by Kenneth I. Ranney, Armin Doerry, Proc. of SPIE Vol. 10188, $1018808 \cdot$ (c) 2017 SPIE · CCC code: 0277-786X/17/\$18 · doi: 10.1117/12.2261680 


\subsection{UWB and Continuous-Wave Based Through-the-Wall Radar}

TW radar systems employing conventional radar transmission waveforms are typically based on either short ultrawideband (UWB) pulses ${ }^{6}$ or longer continuous waves $(\mathrm{CW})^{7,8}$. In addition to the superior range-resolutions which are achievable with UWB waveforms, they are also scattered well by targets of differing scales giving rise to improved target recognition capabilities compared to narrowband systems. Disadvantages though include the high costs of electronic components suitable for UWB radar, including the requirement to have expensive wideband antennas, and their susceptibility to interference from signals in overlapping spectral bands. Impulse radar is special class of UWB radar which has been examined for TW radar applications'; a short nanosecond 'spike-like' pulsed waveform is transmitted which gives a near- $100 \%$ fractional bandwidth for excellent range resolution. However, the extreme bandwidth results in significant noise power entering the receiver which is detrimental to system performance.

Continuous waveforms are characterised as being always 'On', and have many typologies. In their most basic form CW radars transmit a stable frequency continuous wave which allows the application of Doppler processing techniques for sensing moving targets. However they are not able to determine the range to a target and are therefore not typically used in TW radar. Frequency modulated continuous wave (FMCW) radar, where the instantaneous transmission frequency changes linearly across the waveform, overcomes this ranging issue and is now a widely adopted solution for low-cost, short to medium range sensing applications, including TW applications ${ }^{10}$. Key advantages of FMCW radar include good immunity to unwanted signal interference and low peak power and sampling rate requirements. A vehicle-based TW radar system developed at Lincoln $\mathrm{Labs}^{7}$ which combines an FMCW radar architecture with UWB chirp transmission waveforms has been shown to be able to locate both moving and stationary people behind $20 \mathrm{~cm}$ think concrete walls.

Other types of CW radars which have been investigated for TW applications include stepped-CW systems which transmit short $\mathrm{CW}$ bursts at progressively higher frequencies, and frequency hopping radars which have the agility to move their operational frequencies back and forth ${ }^{11}$. Dual frequency waveforms ${ }^{12}$ are a special case of CW-only waveforms which are able to circumvent the ranging issues in conventional $\mathrm{CW}$ radar and have been employed by Amin et $a l^{13}$ TW detection; here two carrier waves of different frequencies are transmitted and on receive the phase changes between them are compared. Recently both pure noise waveforms (sometimes referred to as random noise waveforms) and noise-like waveforms (pseudonoise waveforms) have been tried and tested for TW radar. These waveforms have been designed specifically to optimise range and Doppler resolutions while minimising entities such as sidelobes which act to degrade the quality of the radar output. The main advantages of noise-based waveforms are that they offer good performance whilst having an antijamming capability due to their low probability of detection. Finally, the field of digital communications has also provided a substantial pool of sophisticated continuous waveforms from which to draw from in TW radar research. For example M-sequences offers high-correlation gains and operation at low-power and low$\operatorname{cost}^{14,15}$, while OFDM waveforms used in IEEE 802.11 (WiFi) communications, and which are resistant to multipath, have been exploited by a passive TW radar system ${ }^{16}$ and TW mapping robot for building indoor floor-plans ${ }^{17}$.

\subsection{Through-Wall Activity Recognition}

An emerging area receiving significant interest in TW radar research is the use of micro-Doppler signature analysis to allow a more complete description of the kinematic properties of targets, which can then be used to identify different types of human movements ${ }^{18}$. Micro-Doppler is an additional effect on top of the main 'bulk' Doppler component whereby the motions on the main body of movement, such as rotations (e.g. the swinging limbs of a person) induce further Doppler frequency perturbations around the main Doppler shift. These perturbations, or micro-Doppler signatures, are characteristic to particular types of movement ${ }^{19}$ and can therefore be used to provide extra information about the targets of interest using classification techniques ${ }^{20}$. Ram et $a l^{21}$ Ram et al present a simulation tool for generating micro-Doppler signatures of humans moving behind walls. Their approach entails combining standard human modelling techniques with finite difference time-domain of the wall and is validated by experimental data. The results suggest that although the returned signal power is severely compromised by the wall, the effect on the micro-Doppler signature is minimal.

In this paper we present a low-cost TW FMCW radar for stand-off operation and activity detection. Section 2 outlines the dual-frequency FMCW radar architecture whilst Section 3 describes the de-ramp signal processing (Section 3.1) and demonstrates the utility of the MTI processing to recover target responses that would have otherwise been obscured by the primary wall reflection (Section 3.2). Next in Section 4, results are presented from a series of experimental field trials which focussed on: characterising the detection capability of the radar through brick and interior plasterboard walls; 
detection of multiple targets; and highlighting the potential of the system to perform TW micro-Doppler activity analysis on the recorded data. Finally, Section 5 summarises the main outcomes of the work and future research plans.

\section{SYSTEM DESIGN}

\subsection{System Architecture}

The FMCW radar system was designed and built at University College London (UCL) and operates at $5.8 \mathrm{GHz}$ with a $200 \mathrm{MHz}$ bandwidth. A block diagram of system is illustrated in Figure 1.

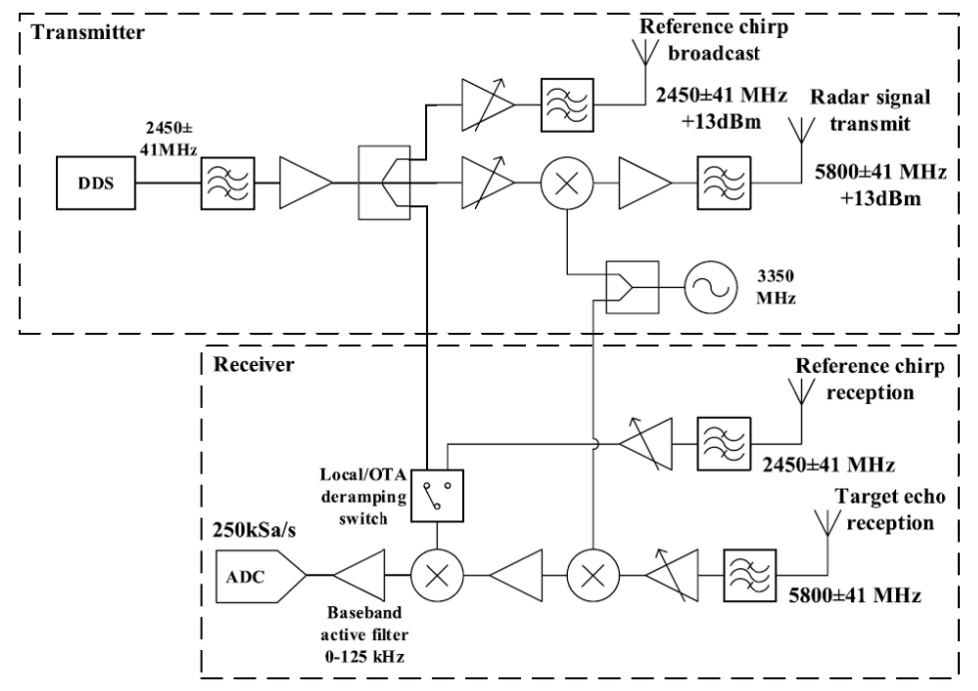

Figure 1. System block diagram of the FMCW through-wall radar

The TW FMCW radar system employs a super-heterodyne architecture. A linear chirp signal is first generated by the direct digital synthesizer (DDS) chip at an intermediate frequency (IF) of $2.45 \mathrm{GHz}$ before being unconverted to its 5.8 $\mathrm{GHz}$ operating frequency. This dual-frequency design minimises interference arising from signal leakage. The maximum transmission power of the radar after bandpass filtering and amplification is $+13 \mathrm{dBm}$ and its noise figure is $2.5 \mathrm{~dB}$. Digitization of the output baseband signal is carried out using a commercial off-the-shelf (COTS) 24-bit external sound card to give high dynamic range at low-cost. The radar has the capability to work with duplicate radar nodes within a bistatic configuration using a technique termed over-the-air-deramping ${ }^{22}$. However, in this TW work only the basic monostatic mode was utilised whereby the reference chirp broadcast and reception paths within the transmit and receiver chains are disabled.

\section{SIGNAL PROCESSING}

\subsection{De-ramp Signal Processing}

The TW FMCW radar continuously transmits a chirp waveform which is of the form:

$$
x_{0}(t)=a_{0} \cos 2 \pi\left[f_{0} t+(1 / 2) \alpha t^{2}\right]
$$

where $a_{\mathrm{o}}$ is the signal amplitude, $f_{\mathrm{o}}$ is the chirp start frequency and $\alpha$ is the chirp rate (the ratio of the chirp bandwidth and the chirp period, $B / T$ ). After being reflected from the target of interest, the echo signal $x_{t}$ returns to the receiver after a propagation delay $\tau$, given by

$$
x_{t}(t)=a_{t} \cos 2 \pi\left[f_{0}\left(t-\tau_{t}\right)+(1 / 2) \alpha\left(t-\tau_{t}\right)^{2}\right]
$$


The target echo signal at $f_{\mathrm{o}}$ is filtered, amplified and translated to an IF equal to $f_{\mathrm{s}}$ before entering the deramp mixer which after low-pass filtering gives a deramped output signal of the following form:

$$
y_{d}(t)=x_{0}(t) \cdot x_{t}(t)=d_{0} \cos 2 \pi\left[f_{0} \tau_{t}+\alpha t \tau_{1}-(1 / 2) \alpha \tau_{t}^{2}\right]
$$

$\alpha \tau 1$ is known as the deramp frequency fd and corresponds to a beat signal whose frequency is proportional to the target range according to equation (4)

$$
f_{d}=\frac{2 B R_{t}}{c T}
$$

\subsection{Primary Wall Suppression using MTI Processing}

Moving Target Indication (MTI) processing is used to remove the return from stationary walls which can be considered as a DC component. MTI processing first involves passing individual received chirps through a hanning window to supress range sidelobes. A finite impulse response (FIR) digital high pass filter is then applied across multiple chirps to enhance moving target responses while minimising the component response of the wall. With the target velocities of a few meters per second being considered it was found a high-order FIR filter performs best as IIR filters become unstable at such low cut-off frequencies The FIR filter is implemented using the window method. Suppose the frequency response is $H(\omega)$, then the impulse response sequence $h[n]$ is given by equation (5)

$$
h[n]=\frac{1}{2 \pi} \int_{-\pi}^{\pi} H(\omega) \times e^{j \omega n} d \omega
$$

Figure 2 demonstrates the utility of our MTI processing through an experiment which involved a person walking backwards and forwards inside a house whilst being monitored by the FMCW radar around $8.5 \mathrm{~m}$ away, and through a $33 \mathrm{~cm}$ air-gapped brick wall. Figure 2a shows a range-time profile of the result prior to the application of our MTI algorithm. The target response (highlighted in the figure) is well below the strong peak generated by the primary wall reflection and unlikely to register as a target detection. However, after application of the MTI processing to supress the effect of the wall, the signal-to-interference (SIR) of the target movement trace significantly increases. Note that in all cases hereon in, the MTI processing has been applied to the recorded radar data.

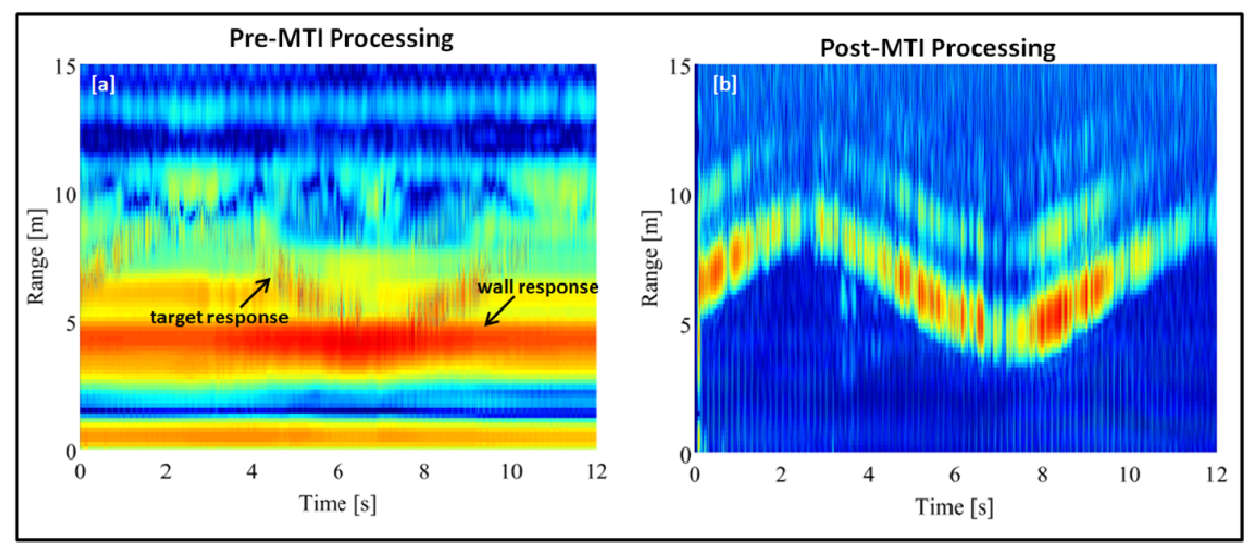

Figure 2. Range versus time plots of for a person walking back and forth inside [a] before and [b] after wall application of our moving target indication (MTI) processing. The bright red response at $4 \mathrm{~m}$ in [a] is caused by the outside wall and masks any other objects in the scene. By applying high-order digital filters in [b], the wall response is removed to reveal the presence of the walking person. 


\section{EXPERIMENTAL RESULTS}

A series of experiments were carried out to characterise the performance of the TW FMCW radar through various wall materials, and investigate the capabilities of the radar when an area of interest contains multiple personnel. To examine the potential of the radar for activity recognition, we also apply phase processing to the recorded data to capture the micro-motions of a person as they simulated everyday actions. In all experiments described in this paper, the radar was configured to continuously transmit $2 \mathrm{~ms}$ up-chirps at the maximum power of $13 \mathrm{dBm}$. Identical $12 \mathrm{dBi}$ gain and $30^{\circ}$ beamwidth antennas were also used which were co-located but separated by a vertical distance of $60 \mathrm{~cm}$.

\subsection{Detection through Plasterboard Walls}

The first experiment investigated the ability of the TW radar system to detect a person through a typical lightweight interior wall made of plasterboard. The thickness of the wall was $12 \mathrm{~cm}$ and consisted of 2 layers of $12.5 \mathrm{~mm}$ thick plasterboard separated by $9 \mathrm{~cm}$, and a wooden frame. The radar was located $1 \mathrm{~m}$ from one side of the wall while a target was located in an adjacent room $9 \mathrm{~m}$ in length. Figure 3 shows a range-time plot for a person walking back and forth along the full length of the room over a period of 20 seconds. Note that due to cable lengths being unaccounted for, there is a $5 \mathrm{~m}$ range offset in the graph.

A temporal trace of the target's motion within the full length of the room can clearly be seen. Moreover, we observe that between $\sim 7-13$ seconds while the target was moving away the radar the SNR degrades, but subsequently increases between $\sim 13-20$ seconds after the target turns around and closes-in on the radar. Analysis of the results suggests a range resolution of $\pm 1.25 \mathrm{~m}$ which is agreement with the systems' operational bandwidth. We also notice some ghosting of the temporal trace which we attribute to multipath caused by reverberations within the air gap of the wall. Note: although the MTI filtering was able to substantially supress the primary wall response, it did not completely remove it and this is highlighted in the figure.

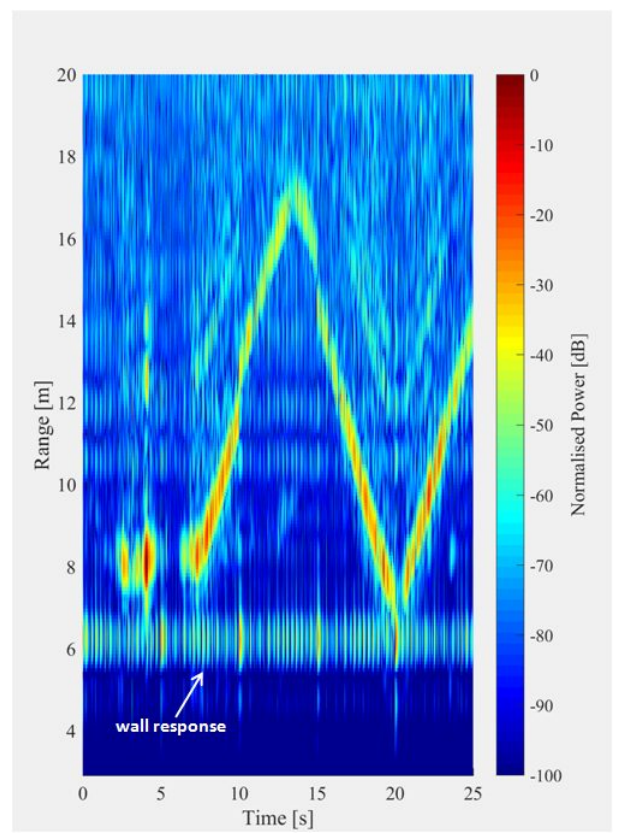

Figure 3. Range-Time plot for a person walking back and forth inside a room of length 9 meters. The obscuring barrier was an internal plasterboard wall.

\subsection{Detection through Brick Walls}

The second series of experiments focussed on a detection scenario in which a $22 \mathrm{~cm}$ thick brick wall was the obscuration barrier. This type of wall represents a standard interior or exterior wall construction. The $11 \mathrm{~m}$ length room in this case 
was divided into 3 areas as illustrated in Figure 4; a close-range zone between 2-5 m from the wall; a mid-range zone between 5-8 m; and a stand-off zone between $8 \mathrm{~m}$ and the end of room at $11 \mathrm{~m}$. As in the first experiment, the radar was located $1 \mathrm{~m}$ away from the wall. Figure 5 shows three range time plots for a person walking forward and backwards within each zone each for a period of approximately 25 seconds. In all cases the target can be detected but again we see the degradation of SNR as a function of radar range. Additionally, in the stand-off zone, the radar reaches its detection limit as target response begins to equal the power of the noise.

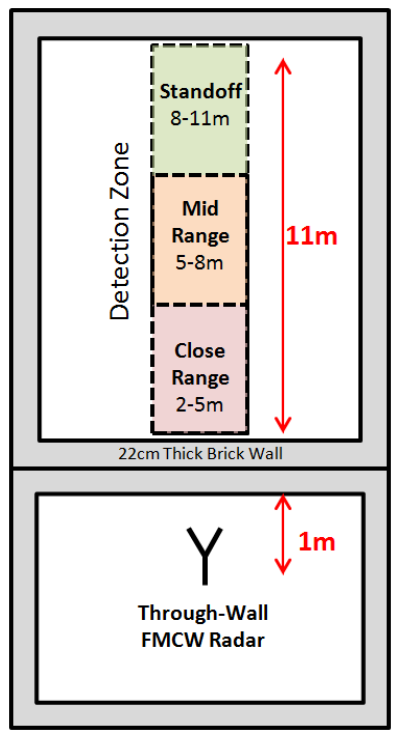

Figure 4. Through-wall detection scenario consisting of a $22 \mathrm{~cm}$ thick brick wall. The range detection capability of the radar was examined using three zones: the close-range zone 2-5 $\mathrm{m}$ away from the brick wall; the mid-range zone between 5-8 $\mathrm{m}$ from the brick wall; and the stand-off zone between 8-11 $\mathrm{m}$ from the brick wall.

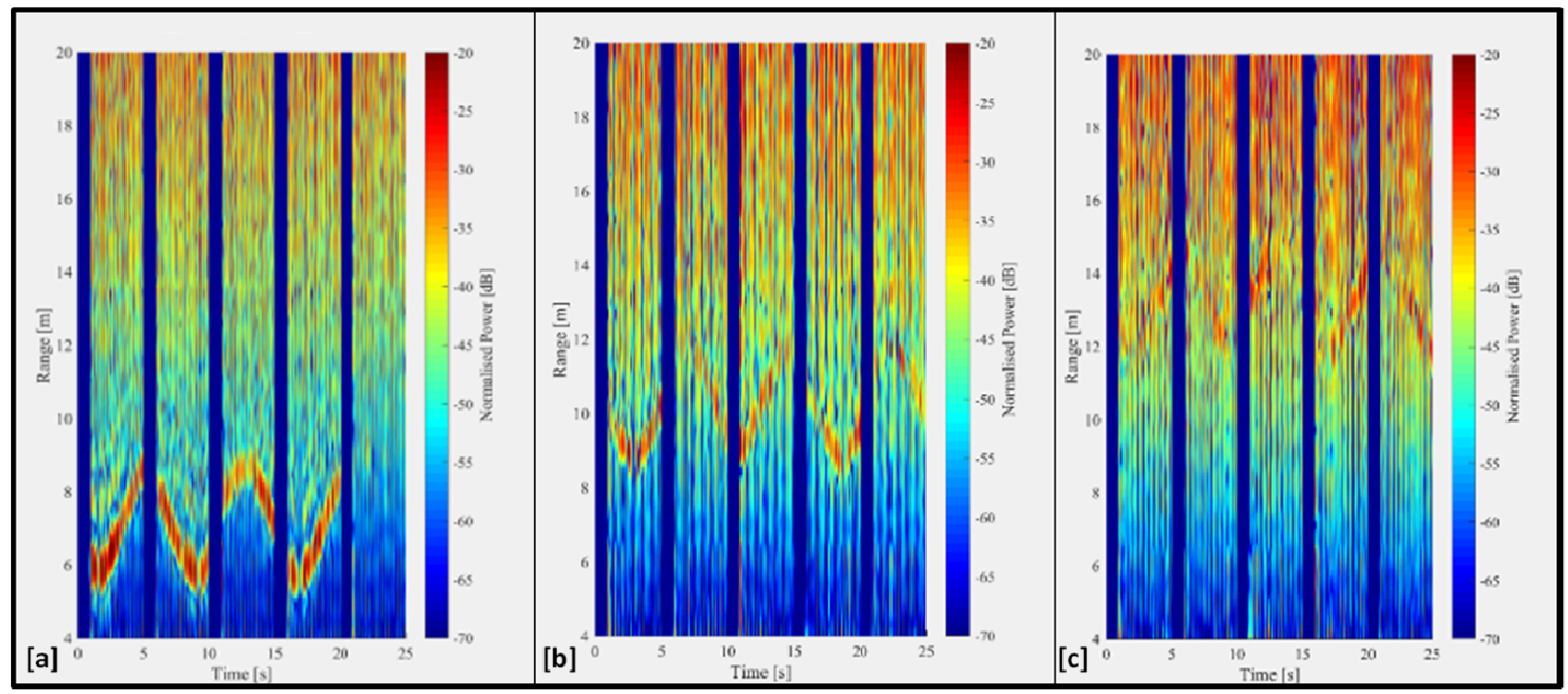

Figure 5. Range-Time plot for a person walking back and forth within [a] the close-range zone; [b] the mid-range zone; and [c] the stand-off zone. 


\subsection{Multiple Target Detection}

Experiment 3 was geared towards investigating the ability of the radar to detect multiple targets within a room of interest. Using the same experimental conditions described in Section 4.1, a number of tests were carried out that involved 2 or more people moving in the $9 \mathrm{~m}$ room. Figure 6a shows the result for a single person walking forward and backwards for a duration of 10 seconds, before being joined by an additional person who attempted to walk in the opposite direction. For the plot in Figure 6b, two people were instructed to walk side-by-side at a normal pace to the wall and back, at this point (approximately 10 seconds in) the first person was told to continue at the same pace, while the second person was asked to speed up. Finally, Figure 6c shows the target detections for three people inside the room. Target 1 was instructed to walk backward and forward in a small area, while Targets 2 and 3 were directed to walk around in a random fashion which meant that they indiscriminately entered and exited the antenna coverage area.
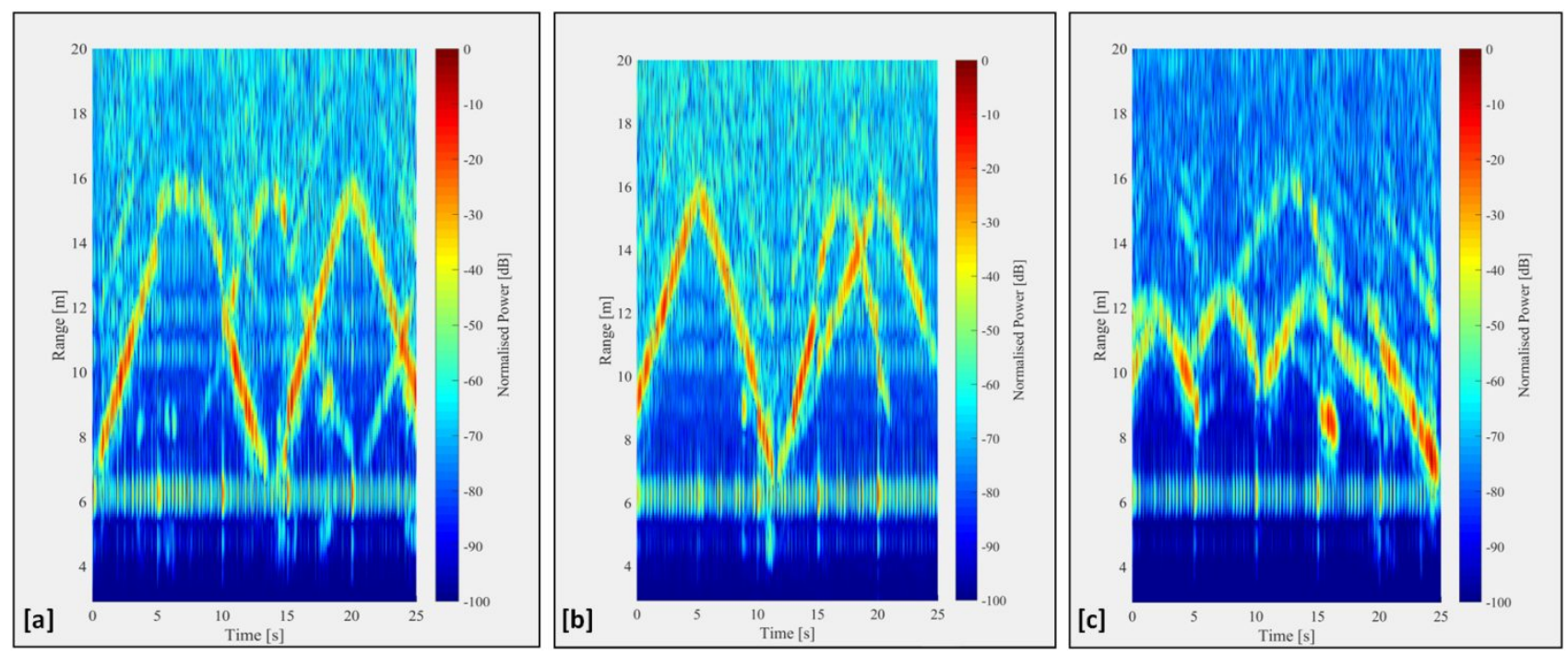

Figure 6. Detection of multiple targets through an interior plasterboard wall. In [a] two people walk in opposite directions; in [b] two people walk side-by-side for 10 seconds before one person speeds up; and in [c] one person walks forwards and backwards while two people move around randomly in and out of the antenna coverage area.

For all the multi-target experiments, the range-time plots successfully indicate the movement dynamics within the scenario. However, in various portions of the graphs (for example Figure $6 \mathrm{~b}$ at $\sim 15$ seconds) shielding occurs between multiple targets which acts to supress echoes below the noise floor.

\subsection{Micro-Doppler Signature Characterisation}

In the final experiment we examine the potential of the radar to distinguish between different types of micro-Doppler signatures, and thus exploit the data for activity classification in the future. Here, a personnel target was monitored through a $33 \mathrm{~cm}$ brick-walled house consisting of a central air-gap. The TW FMCW radar was located outside $43 \mathrm{~cm}$ from the wall. The subject was instructed to remain in the same position whilst performing four actions repeatedly for a duration of 5 seconds each. The four actions were (i) swinging both arms (ii) swaying back and forth (rocking) (iii) squatting into a hiding position from standing and (iv) standing-up from a bended knee position. The recorded data underwent spectrogram analysis to generate velocity-time graphs that reveal micro-Doppler signatures, and these are shown in Figure 7. 


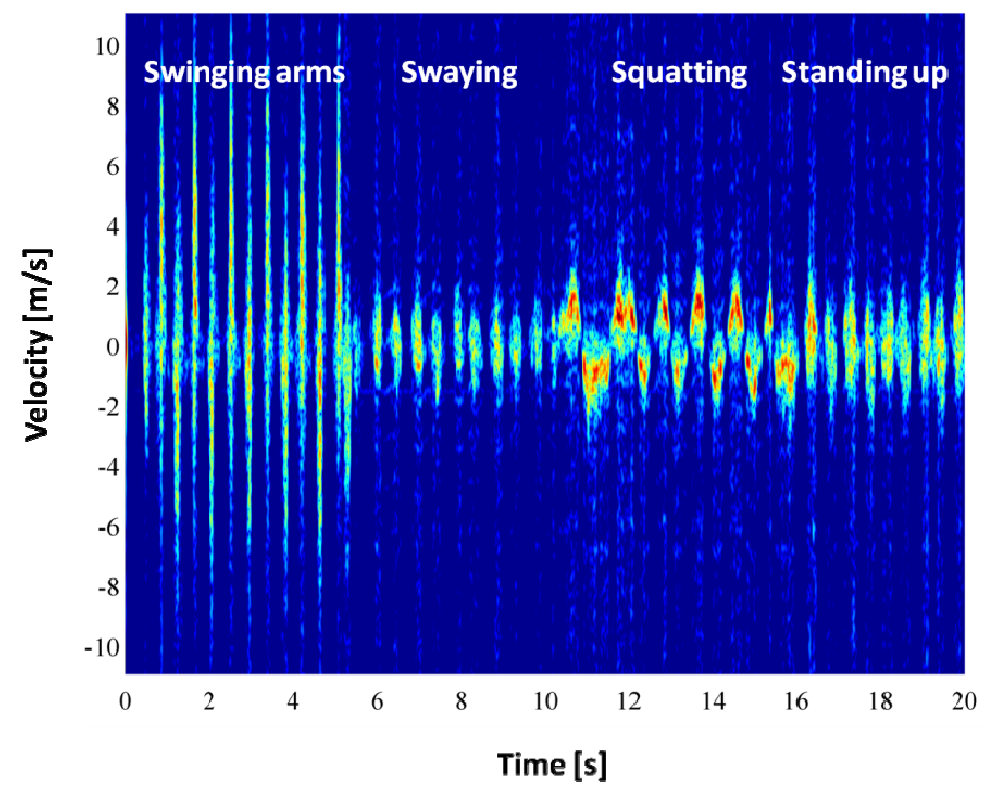

Figure 7. Velocity-time graph illustrating different through-wall micro-Doppler signatures as a person performed for everyday actions. Each action was repeated for approximately 5 seconds.

The velocity-time patterns displayed in Figure 7 indicate clear differences in the micro-Doppler signatures between the four types of motions. Although this is only a preliminary experiment, it highlights the potential for carrying out throughwall activity recognition using micro-Doppler classification techniques [18].

\section{SUMMARY AND FUTURE WORK}

In this paper we present a $5.8 \mathrm{GHz}$ TW FMCW radar system that is an order of magnitude lower in cost that competitor TW systems, owing to a system architecture that allows low-cost COTS components to be utilised. The high sensitivity and dynamic range afforded using a 24-bit external sound card enables incorporation of a wall-removal algorithm in the post-processing based on high-order FIR filtering. This algorithm effectively suppresses of the primary wall reflection, permitting the recovery of target responses that would have otherwise been masked by its large reflection.

A measurement campaign has been carried out using walls of different constructions representative of typical internal and external structures. Scenarios involving both individual and multiple personnel targets were also investigated. It is found that for interior plasterboard walls, targets can be detected as far out as $10 \mathrm{~m}$ (radar to target distance) which could be further increased in a larger room. For $22 \mathrm{~cm}$ thick brick walls, the detection limit is around the $11 \mathrm{~m}$ region. Scenarios in which 2 and 3 personnel targets were investigated also demonstrated the ability of the radar to resolve their positions when separated by more than $1.25 \mathrm{~m}$. Finally, an initial examination of recorded responses using phase processing whilst the target underwent everyday activities such as squatting and standing-up revealed characteristically difference micro-Doppler signatures. Future work will focus on applying target classification techniques to a larger set of measured micro-Doppler data to examine the utility of the FMCW radar for TW activity recognition. Moreover, we plan to further enhance the TW situational awareness capability of the radar by using multiple nodes to fuse data from numerous look-angles; this will be achieved by exploiting the radar's over-the-air deramping function.

\section{REFERENCES}

[1] D. L. Kyllo, [Kyllo, Petitioner v. United States], (2001).

[2] M. Amin, and K. Sarabandi, "Special Issue on Remote Sensing of Building Interior," Geoscience and Remote Sensing, IEEE Transactions on, 47(5), 1267-1268 (2009).

[3] M. Amin, and R. N. Madan, “Editorial,” Journal of the Franklin Institute, 345(6), 553-555 (2008). 
[4] M. I. Skolnik, [Introduction to Radar Systems] McGraw-Hill, (1980).

[5] M. Amin, [Through-the-Wall Radar Imaging] CRC Press, (2011).

[6] R. J. Fontana, "Recent system applications of short-pulse ultra-wideband (UWB) technology," Microwave Theory and Techniques, IEEE Transactions on, 52(9), 2087-2104 (2004).

[7] J. E. Peabody Jr, G. L. Charvat, J. Goodwin et al., "Through-Wall Imaging Radar,” Lincoln Laboratory Journal, 19(1), (2012).

[8] F. Fioranelli, S. Salous, I. Ndip et al., "Through-The-Wall Detection With Gated FMCW Signals Using Optimized Patch-Like and Vivaldi Antennas," Antennas and Propagation, IEEE Transactions on, 63(3), 11061117 (2015).

[9] G. Barrie, [Technical Report - UWB Impulse Radar Characterization and Processing Techniques], (2004).

[10] N. Maaref, P. Millot, C. Pichot et al., "A Study of UWB FM-CW Radar for the Detection of Human Beings in Motion Inside a Building," Geoscience and Remote Sensing, IEEE Transactions on, 47(5), 1297-1300 (2009).

[11] A. R. Hunt, "Use of a Frequency-Hopping Radar for Imaging and Motion Detection Through Walls," Geoscience and Remote Sensing, IEEE Transactions on, 47(5), 1402-1408 (2009).

[12] W. D. Boyer, “A Diplex, Doppler Phase Comparison Radar,” Aerospace and Navigational Electronics, IEEE Transactions on, ANE-10(1), 27-33 (1963).

[13] P. Setlur, M. Amin, and F. Ahmad, "Dual-frequency Doppler radars for indoor range estimation: Cramer-Rao bound analysis," Signal Processing, IET, 4(3), 256-271 (2010).

[14] M. Aftanas, E. Zaikov, M. Drutarovsky et al., "Throughwall imaging of the objects scanned by M-sequence UWB radar system." 1-4.

[15] P. K. Kumar, and T. Kishore Kumar, "M-Sequence UWB radar for three dimensional through-the-wall imaging." 132-137.

[16] K. Chetty, G. E. Smith, and K. Woodbridge, "Through-the-Wall Sensing of Personnel Using Passive Bistatic WiFi Radar at Standoff Distances," Geoscience and Remote Sensing, IEEE Transactions on, 50(4), 1218-1226 (2012).

[17] B. Tan, K. Chetty, and K. Jamieson, [ThruMapper: Through-Wall Building Tomography with a Single Mapping Robot] ACM, Sonoma, CA, USA (2017).

[18] V. C. Chen, D. Tahmoush, and W. Miceli, [Micro-Doppler Signatures - Review, Challenges, and Perspectives] Institution of Engineering and Technology, (2014).

[19] G. E. Smith, K. Woodbridge, C. J. Baker et al., "Multistatic micro-Doppler radar signatures of personnel targets," Signal Processing, IET, 4(3), 224-233 (2010).

[20] A. Balleri, K. Chetty, and K. Woodbridge, "Classification of personnel targets by acoustic micro-doppler signatures," Radar, Sonar \& Navigation, IET, 5(9), 943-951 (2011).

[21] S. S. Ram, C. Christianson, K. Youngwook et al., "Simulation and Analysis of Human Micro-Dopplers in Through-Wall Environments," Geoscience and Remote Sensing, IEEE Transactions on, 48(4), 2015-2023 (2010).

[22] M. Ash, M. Ritchie, K. Chetty et al., "A New Multistatic FMCW Radar Architecture by Over-the-Air Deramping,” IEEE Sensors Journal, 15(12), 7045-7053 (2015). 